\title{
Application of Stream Function Method in Local Route Planning for UUVs
}

\author{
Xinyong Wang ${ }^{1}$, Yang $\mathrm{Ju}^{2, a}$ \\ ${ }^{1}$ Systems Engineering Research Institute, Beijing, China \\ ${ }^{2}$ Science and Technology on Underwater Acoustic Antagonizing Laboratory, Beijing, China \\ ajyang-pioneer@163.com
}

Keywords: Stream function, Local route planning, Maneuver constraints, Detection constraints, Obstacle of complex shape

\begin{abstract}
The research of UUV route planning is becoming an important topic nowadays. In this paper, stream function method coming from fluid dynamics is presented, and it is applicable in the field of local route planning research. In addition, in terms of two problems in application considering maneuver and detection constraints, the improved stream function method are proposed which is the effective solution for the problems.
\end{abstract}

\section{Introduction}

Unmanned underwater vehicles, referred UUV, are highly valued by all combat forces as an emerging military power. Route planning for UUV is one of the core issues in the field of research. For avoiding the dynamic obstacles appearing in the planned path, sensor information is needed for local route planning, which is becoming an important research topic for UUVs.

UUV local route planning is a NP-Hard problem [1]. The traditional optimization methods, such as gradient method, enumeration method, random search method etc., will have lack of robustness, high computational complexity and other problems. In recent decades using tabu search, simulated annealing, genetic algorithms, neural networks and other developed modern optimization methods can solve some practical problems, but still fall into local minimum in certain time.

Stream function method is a new algorithm arising in the field of route planning in recent years. By drawing fluid mechanics concepts, it establishes the potential field, simulates flow mechanism, so that the planned route can go around obstacles smoothly as the water avoid the rocks in the river, which is appears to solve the local minima problems. This method is applied in the fields of environment, metallurgy etc. [2] before, and it is still new in UUVs. It is proposed a UUV local route planning algorithm based on stream function method, and for some specific problems from the practical application, the corresponding solutions are also proposed.

\section{The UUV local route planning algorithm based on stream function method}

Algorithm Design. The UUV local route planning algorithm with stream function [3-5] is based on the basic concepts of fluid mechanics, and the circle theorem is applied, the key steps are:

1) There is a coordinate system in the planning plane XOY, and define the starting point I and the destination point $T$.

2) In the point T, a complex potential $f_{s}(z)$ is added to the meeting point, $f_{s}(z)=-C \ln (z)$ (C is flow field strength). The stream function is $\psi=-C \arctan \frac{y}{x}$.

3) A circular obstacle $o\left(o_{x}, o_{y}\right)$, radius $\mathrm{r}$, and the flow field complex potential

$$
\tilde{f}_{s}(z)=-C \ln (z)-C \ln \left(\frac{r^{2}}{z-o}+\bar{o}\right),
$$

where $o=o_{x}+i o_{y}$, and the stream function is 


$$
\tilde{\psi}=-C \arctan \frac{y}{x}+C \arctan \frac{r^{2}\left(y-o_{y}\right)+o_{y}\left(\left(x-o_{x}\right)^{2}+\left(y-o_{y}\right)^{2}\right)}{r^{2}\left(x-o_{x}\right)+o_{x}\left(\left(x-o_{x}\right)^{2}+\left(y-o_{y}\right)^{2}\right)},
$$

its velocity component is $\mathrm{u}$ and $\mathrm{v}$ in the $\mathrm{x}$ and $\mathrm{y}$-direction, respectively

$$
\begin{aligned}
& u=\frac{\partial \tilde{\psi}}{\partial y}=\frac{-C x}{x^{2}+y^{2}}+C \frac{\left(r^{2}+2 o_{y}\left(y-o_{y}\right)\right) m-2 n o_{x}\left(y-o_{y}\right)}{m^{2}+n^{2}}, \\
& v=-\frac{\partial \tilde{\psi}}{\partial x}=\frac{-C y}{x^{2}+y^{2}}-C \frac{2 m o_{y}\left(x-o_{x}\right)-n\left(r^{2}+2 o_{x}\left(x-o_{x}\right)\right)}{m^{2}+n^{2}},
\end{aligned}
$$

where $m=r^{2}\left(y-o_{y}\right)+o_{y}\left(\left(x-o_{x}\right)^{2}+\left(y-o_{y}\right)^{2}\right), n=r^{2}\left(x-o_{x}\right)+o_{x}\left(\left(x-o_{x}\right)^{2}+\left(y-o_{y}\right)^{2}\right)$.

For multiple obstacles, their velocity component is derived from the weighted sum of velocity of each obstacle.

4) Solving the ordinary differential equations $\left\{\begin{array}{l}x^{\prime}=u \\ y^{\prime}=v\end{array}\right.$, the flow line equation $L(x, y)$ is available.

The flow line $L(\mathrm{~T})$ which is right through the destination point $\mathrm{T}$ is desire.

The determine principles of obstacle parameters. In most circumstances, the actual obstacles in the planning plane have irregular shape. To deserve the center and radius of each obstacle, the principles below have to be followed. Suppose the cross section of the obstacle is irregular shape in the planning planeXOY. From the start point I side, according to the real-time information collected from the sensors, the maximum distance between any of two points on the edge of the cross-section of obstacles can be obtained. Then according to the minimum distance point $\mathrm{K}$ away from the straight line of the starting point, the vertical distance $b$ is measurable, and the other measurable distances are shown in Fig. 1. Hence, the midpoint of PQ is taken to be the center of the obstacle, and the maximum length of $\mathrm{OQ}, \mathrm{OK}$ is taken to be the radius of the obstacle, i.e.,

$$
r_{1}=\frac{1}{2}|P Q|=\frac{1}{2} \sqrt{(c-a)^{2}+d^{2}}, \quad r_{2}=|O K|=\sqrt{\left(\frac{a+c}{2}-b\right)^{2}+e^{2}}, \quad r=\max \left(r_{1}, r_{2}\right) .
$$

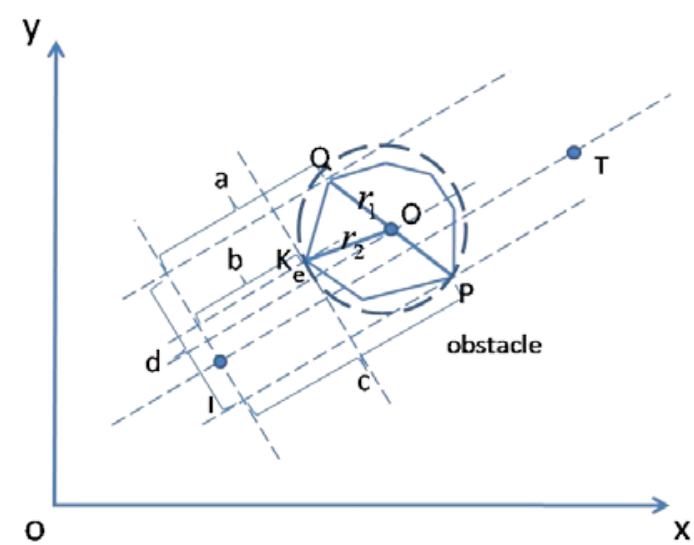

Fig. 1 Parameters decision of obstacle

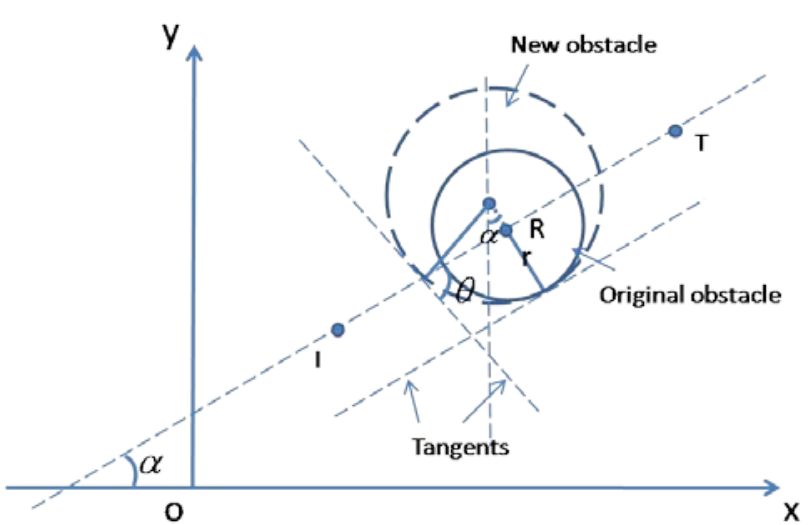

Fig. 2 Virtual obstacle setting

\section{The improved UUV local route planning algorithm}

In practice, with constraints of the performance of maneuver and detection of UUV and the shape characteristics of obstacles, the performance of local route planning algorithm based on stream function method will become less powerful, and in some cases it may even fail. The improved methods for two typical cases are proposed as follows.

The maneuver constraint of UUV. In the application of UUV local route planning algorithm with stream function, the problem can arise when the line connected the starting point and the 
destination point or the line is not far from the center. When the line across the center, the "stagnant" phenomenon will be happened, ie. the route is interrupted on obstacle edge, which causes algorithm failure; when the line is not far from the center, the large turning angle is needed to go around obstacles, and if the turning angle exceeds the maximum maneuvering angle of UUV, the route will also fail. To avoid the two problems, the new determine principles of obstacle parameters are established.

In the planning plane XOY, given the center $\left(x_{0}, y_{0}\right)$, radius $\mathrm{r}$ of original obstacle, the maximum turning angle $\theta$ of UUV, and the angle $\alpha$ the line connected the starting point and the destination point with the x-axis (Fig. 2). Suppose the new center of obstacles is $\left(x_{N}, y_{N}\right)$, and the new radius is R, then

$$
R=\frac{r}{1-\cos \theta}, \quad y_{N}=y_{0}+(R-r) \cos \alpha, \quad x_{N}=x_{0}-(R-r) \sin \alpha
$$

Thus, local route planning with the new parameters of obstacle can avoid stagnation, and meet the maneuver requirements of UUVs.

The detection performance constrain of UUV. Typically, the detection capability of underwater sensor is always limited. Based on the real-time information only provided by forward looking sonar the morphological characteristics of the obstacle do not often be pictured, causing UUV circumvent unsuccessfully. On the other hand, even if we can learn the specific shape of the obstacle through other sources of information, the route would be not satisfied the requirements of the shortest path in accordance with the traditional local route planning. Therefore, we adopt the means that real-time updates the shape and location of obstacles, cut the original obstacles into several sections, plan the route segment of each section, and the optimization route is taken to be the connection of every segment. The core of the improved algorithm is still to determine the center and radius of each obstacle.

In the planning plane XOY, given an irregular rectangular shape obstacle (Fig. 3), when UUV reached point $I_{1}$ by the path along the route planned from the obstacle $O_{1}$, there is a new obstacle appears through the sensor information, which with the nearest length from point $I_{1}$ at point $\mathrm{P}$, and with the maximum distance from the straight $I_{0} T_{0}$ at point $\mathrm{Q}$. The route optimization steps are:

1) Make point $I_{1}$ to be the sub-starting point in the next phase;

2) Make sub-destination point to be always selected properly from the line $I_{0} T_{0}$ between the original starting point $I_{0}$ and destination point $T_{0}$;

3) Make the region which is get from removing the obstacle $O_{1}$ from the visible obstacle at point $I_{1}$ to be the new obstacle;

4) Make the new center of obstacle $O_{2}$ to be always taken on the line through point $O_{1}$ and parallel to the straight line $I_{0} T_{0}$, and make $\left|O_{2} Q\right|$ to be the radius of the obstacle $O_{1}$;

5) The radius of the new obstacle is $r_{2}=\max \left(\left|O_{2} Q\right|,\left|O_{2} P\right|\right)$;

6) Analyze the information of visible area of obstacle when UUV sailing at the point $I_{2}$, and determine whether to enter the next phase. If so, return to step 1 ; if not, route planning is completed.

For example, given the center of the obstacle $O_{1}\left(x_{1}, y_{1}\right)$, radius $r_{1}, P\left(x_{P}, y_{P}\right), Q\left(x_{q}, y_{q}\right)$, the distance $\mathrm{d}$ from the point Q to the straight line $O_{1} O_{2}$, and the angle $\alpha$ of the line $I_{0} T_{0}$ against the $\mathrm{x}$-axis. Suppose the center of the obstacle $O_{2}$ as $(x, y)$, the radius as $r_{2}$, and the angle of $O_{2} Q$ with the $x$-axis as $\beta$, then

$$
\beta=\frac{\pi}{2}-\alpha-\arccos \frac{d}{r_{1}}, x=x_{1}-r_{1} \cos \beta, y=y_{1}-r_{1} \sin \beta, r_{2}=\max \left(r_{1}, \sqrt{\left(x-x_{p}\right)^{2}+\left(y-y_{p}\right)^{2}}\right),
$$

Thus, along two route segments planned from $I_{0} T_{0}$ and $I_{1} T_{1}$, the obstacles can be avoided successfully. 


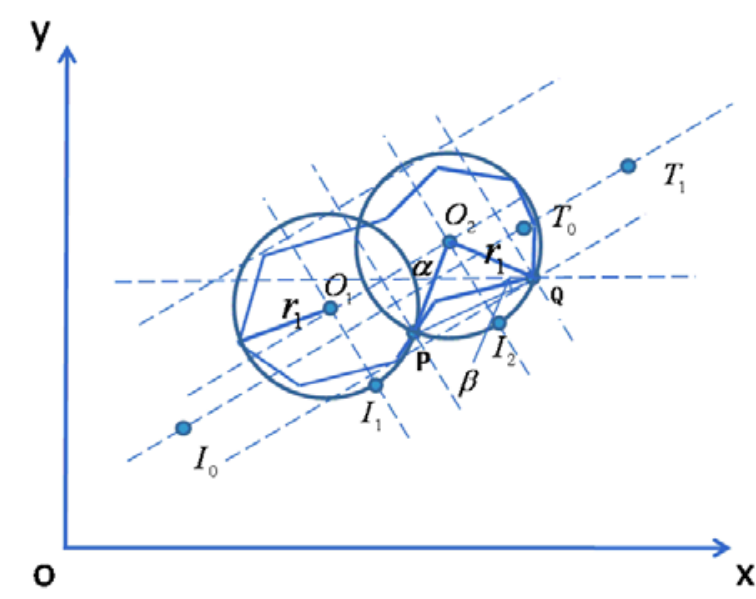

Fig. 3 Virtual Destination point setting

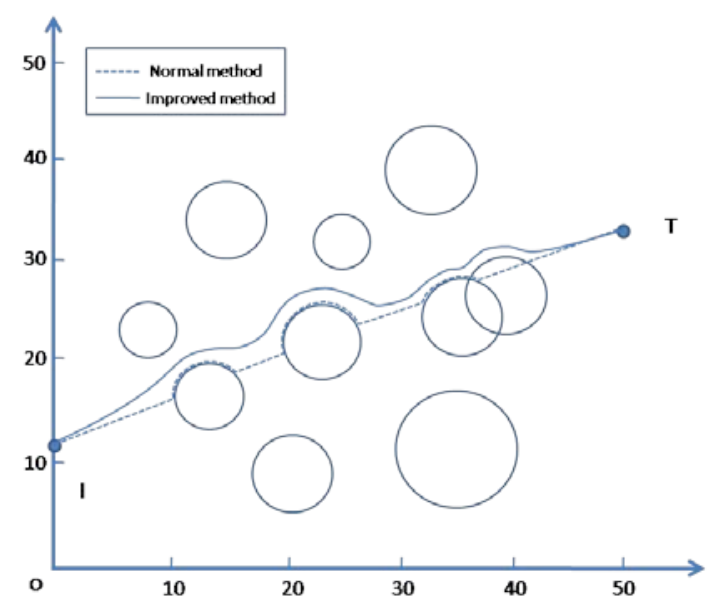

Fig. 4 The simulation results

\section{Experiments}

Assuming UUV sailing in the $50 * 50$ planning plane, analog cross-section of obstacles with a rotary parabolic, and set 10 obstacles to simulate the situations more complex: one situation is the line connected the starting point I and the destination point $\mathrm{T}$ comes across the center of obstacles, and the other is two obstacles overlapping. Assuming maximum maneuvering angle of UUV is $60^{\circ}$, using the normal method and the improved method with stream function to plan the route, respectively, which is shown in Fig. 4. As can be seen, in both complex situations, the route from the improved method can right round obstacles with a small curvature.

\section{Conclusions}

It is presented the local route planning algorithm based on stream function method and its improved algorithm against the restrictions of maneuvering and detection of UUV. In this paper, the effect of underwater environment is not taken into account. In practice, if the water environment has impact of the potential in the flow field, it will affect the final result of path planning. Therefore, it needs further study and improve on whether the existence of these effects and how it affects.

\section{References}

[1] Zhuang Xiaobo, Qi Liang. Research on a method for high speed unmanned ship to avoid collision with moving obstacles. Ship Electronic Engineering, 2008,28(12): 95-97, 117.

[2] Wang Zhenfen, Manabu Kiuchi. Application of 3D fluxional function to metal forming. Journal of Harbin Institute of Technology, 2000,32(5): 93-96.

[3] White F M, Fluid mechanics (5th ed.). New York: McGraw-Hill, 2003.

[4] John D. Anderson. Computational fluid dynamics. Wu Songping, Liu Zhaomiao. Beijing: China Machine Press, 2007.

[5] WAYDO S, MURRAY R M. Vehicle motion planning using stream functions. Proceedings of the IEEE International Conference on Robotics and Automation, Taipei, Taiwan: IEEE Computer Society Press, 2003:2484-2491. 